\title{
Space and laboratory discovery of $\mathrm{HC}_{3} \mathrm{~S}^{+\star}$
}

\author{
J. Cernicharo ${ }^{1}$, C. Cabezas ${ }^{1}$, Y. Endo ${ }^{2}$, N. Marcelino ${ }^{1}$, M. Agúndez ${ }^{1}$, B. Tercero ${ }^{3,4}$, J. D. Gallego ${ }^{4}$, and P. de Vicente ${ }^{4}$ \\ 1 Grupo de Astrofísica Molecular, Instituto de Física Fundamental (IFF-CSIC), C/ Serrano 121, 28006 Madrid, Spain \\ e-mail: jose.cernicharo@csic.es \\ 2 Department of Applied Chemistry, Science Building II, National Chiao Tung University, 1001 Ta-Hsueh Rd., Hsinchu 30010, \\ Taiwan \\ 3 Observatorio Astronómico Nacional (IGN), C/ Alfonso XII, 3, 28014 Madrid, Spain \\ 4 Centro de Desarrollos Tecnológicos, Observatorio de Yebes (IGN), 19141 Yebes, Guadalajara, Spain
}

Received 27 November 2020 / Accepted 9 January 2021

\begin{abstract}
We report the detection in TMC-1 of the protonated form of $\mathrm{C}_{3} \mathrm{~S}$. The discovery of the cation $\mathrm{HC}_{3} \mathrm{~S}^{+}$was carried through the observation of four harmonically related lines in the $Q$ band using the Yebes $40 \mathrm{~m}$ radiotelescope, and is supported by accurate ab initio calculations and laboratory measurements of its rotational spectrum. We derive a column density $N\left(\mathrm{HC}_{3} \mathrm{~S}^{+}\right)=(2.0 \pm 0.5) \times 10^{11} \mathrm{~cm}^{-2}$, which translates to an abundance ratio $\mathrm{C}_{3} \mathrm{~S} / \mathrm{HC}_{3} \mathrm{~S}^{+}$of $65 \pm 20$. This ratio is comparable to the $\mathrm{CS} / \mathrm{HCS}^{+}$ratio $(35 \pm 8)$ and is a factor of about ten larger than the $\mathrm{C}_{3} \mathrm{O} / \mathrm{HC}_{3} \mathrm{O}^{+}$ratio previously found in the same source. However, the abundance ratio $\mathrm{HC}_{3} \mathrm{O}^{+} / \mathrm{HC}_{3} \mathrm{~S}^{+}$ is $1.0 \pm 0.5$, while $\mathrm{C}_{3} \mathrm{O} / \mathrm{C}_{3} \mathrm{~S}$ is just $\sim 0.11$. We also searched for protonated $\mathrm{C}_{2} \mathrm{~S}$ in TMC- 1 , based on ab initio calculations of its spectroscopic parameters, and derive a $3 \sigma$ upper limit of $N\left(\mathrm{HC}_{2} \mathrm{~S}^{+}\right) \leq 9 \times 10^{11} \mathrm{~cm}^{-2}$ and a $\mathrm{C}_{2} \mathrm{~S} / \mathrm{HC}_{2} \mathrm{~S}^{+} \geq 60$. The observational results are compared with a state-of-the-art gas-phase chemical model and conclude that $\mathrm{HC}_{3} \mathrm{~S}^{+}$is mostly formed through several pathways: proton transfer to $\mathrm{C}_{3} \mathrm{~S}$, reaction of $\mathrm{S}^{+}$with $c-\mathrm{C}_{3} \mathrm{H}_{2}$, and reaction between neutral atomic sulfur and the ion $\mathrm{C}_{3} \mathrm{H}_{3}^{+}$.
\end{abstract}

Key words. astrochemistry - line: identification - ISM: molecules - ISM: individual objects: TMC-1 - molecular data

\section{Introduction}

The cold dark core TMC-1 presents an interesting carbon-rich chemistry that leads to the formation of long neutral carbonchain radicals and their anions (see Cernicharo et al. 2020a and references therein). The carbon chains $\mathrm{C}_{2} \mathrm{~S}$ and $\mathrm{C}_{3} \mathrm{~S}$ are particularly abundant in this cloud (Saito et al. 1987; Yamamoto et al. 1987), as they also are in the carbon-rich circumstellar envelope IRC +10216 (Cernicharo et al. 1987). TMC-1 is also peculiar for the presence of protonated species of abundant carbon chains, such as $\mathrm{HC}_{3} \mathrm{NH}^{+}$(Kawaguchi et al. 1994), $\mathrm{HC}_{3} \mathrm{O}^{+}$ (Cernicharo et al. 2020a), and $\mathrm{HC}_{5} \mathrm{NH}^{+}$(Marcelino et al. 2020). The abundance ratio between a protonated molecule and its neutral counterpart, $\left[\mathrm{MH}^{+}\right] /[\mathrm{M}]$, is sensitive to the degree of ionisation, and therefore also to various physical parameters of the cloud, as well as to the formation and destruction rates of the cation (Agúndez et al. 2015). It is interesting to note that both chemical models and observations suggest a trend in which the abundance ratio $\left[\mathrm{MH}^{+}\right] /[\mathrm{M}]$ increases with increasing proton affinity of M (Agúndez et al. 2015). Thus, protonated species of abundant molecules with high proton affinities are good candidates for detection. This is the case for $\mathrm{HC}_{3} \mathrm{~S}^{+}$, the sulphur analogue of $\mathrm{HC}_{3} \mathrm{O}^{+}$, given that $\mathrm{C}_{3} \mathrm{~S}$ has a very high proton affinity of $933 \mathrm{~kJ} \mathrm{~mol}^{-1}$ (Hunter \& Lias 1998), and is around seven times more abundant than $\mathrm{C}_{3} \mathrm{O}$.

\footnotetext{
* Based on observations carried out with the Yebes $40 \mathrm{~m}$ telescope (projects 19A003, 20A014, and 20D015). The $40 \mathrm{~m}$ radiotelescope at Yebes Observatory is operated by the Spanish Geographic Institute (IGN, Ministerio de Transportes, Movilidad y Agenda Urbana).
}

In this Letter, we report the detection of four harmonically related lines that belong to a molecule with a ${ }^{1} \Sigma$ ground electronic state towards the cold dark core TMC-1. From the derived rotational and distortion constants we conclude, based on detailed ab initio calculations, that the best possible carrier is $\mathrm{HC}_{3} \mathrm{~S}^{+}$, the protonated form of $\mathrm{C}_{3} \mathrm{~S}$. We succeeded in producing this cation in the laboratory and measured its microwave spectrum, which fully confirms our assignment. Previous laboratory studies of this species were performed by Thorwirth et al. (2020) who measured its vibrational spectrum through infrared observations at low spectral resolution. We present a detailed observational study of protonated S-bearing carbon chains in this cloud and discuss these results in the context of a state-of-the-art gasphase chemical model.

\section{Observations}

New receivers built within the Nanocosmos project ${ }^{1}$ and installed at the Yebes $40 \mathrm{~m}$ radiotelescope were used for observations of TMC-1. The $Q$-band receiver consists of two HEMT cold amplifiers covering the $31.0-50.3 \mathrm{GHz}$ band with horizontal and vertical polarizations. Receiver temperatures vary from $22 \mathrm{~K}$ at $32 \mathrm{GHz}$ to $42 \mathrm{~K}$ at $50 \mathrm{GHz}$. The spectrometers are $2 \times 8 \times 2.5 \mathrm{GHz}$ FFTs with a spectral resolution of $38.15 \mathrm{kHz}$ providing the whole coverage of the $Q$-band in both polarisations. The main beam efficiency varies from 0.6 at $32 \mathrm{GHz}$ to 0.43 at $50 \mathrm{GHz}$. A detailed description of the system is given by Tercero et al. (2021).

\footnotetext{
1 https://nanocosmos.iff.csic.es/
} 
The line survey of TMC-1 $\left(\alpha_{J 2000}=4^{\mathrm{h}} 41^{\mathrm{m}} 41 \mathrm{~s} .9\right.$ and $\delta_{J 2000}=$ $+25^{\circ} 41^{\prime} 27^{\prime \prime}(0)$ in the $Q$-band was performed in several sessions. Previous results on the detection of $\mathrm{C}_{3} \mathrm{~N}^{-}$and $\mathrm{C}_{5} \mathrm{~N}^{-}$ (Cernicharo et al. 2020b), $\mathrm{HC}_{5} \mathrm{NH}^{+}$(Marcelino et al. 2020), $\mathrm{HC}_{4} \mathrm{NC}$ (Cernicharo et al. 2020c), and $\mathrm{HC}_{3} \mathrm{O}^{+}$(Cernicharo et al. 2020a) were based on two runs performed in November 2019 and February 2020. In these runs, two different frequency coverages were observed, $31.08-49.52 \mathrm{GHz}$ and $31.98-50.42 \mathrm{GHz}$, which allow us to check that no spurious ghosts are produced in the down-conversion chain in which the signal coming from the receiver is down-converted to $1-19.5 \mathrm{GHz}$, and then split into eight bands of $2.5 \mathrm{GHz}$, each of which are analyzed by the FFTs. Additional data were taken in October and December 2020 to improve the line survey at some frequencies, and to further check the consistency of all observed spectral features.

The observing procedure was frequency-switching with a frequency throw of $10 \mathrm{MHz}$ for the two first runs and of $8 \mathrm{MHz}$ for those of October and December 2020. The intensity scale, antenna temperature $\left(T_{\mathrm{A}}^{*}\right)$, was calibrated using two absorbers at different temperatures and the atmospheric transmission model ATM (Cernicharo 1985; Pardo et al. 2001). Calibration uncertainties have been adopted to be $10 \%$. The nominal spectral resolution of $38.15 \mathrm{kHz}$ was used for the final spectra. The sensitivity varies along the $Q$-band between 0.5 and $2.5 \mathrm{mK}$, which considerably improves previous line surveys in the $31-50 \mathrm{GHz}$ frequency range (Kaifu et al. 2004). All data were analysed using the GILDAS package ${ }^{2}$.

\section{Results}

Line identification in our survey of TMC-1 was performed using the MADEX catalogue (Cernicharo et al. 2012), the Cologne Database of Molecular Spectroscopy catalogue (CDMS; Müller et al. 2005), and the JPL catalogue (Pickett et al. 1998). Among the unidentified lines in our survey we found four lines in nearly perfect harmonic relation 6:7:8:9. The lines are shown in Fig. 1 and the derived line parameters are given in Table 1 . The rotational and distortion constants derived from a fit to these lines are $B=2735.4630 \pm 0.0012 \mathrm{MHz}$ and $D=0.171 \pm 0.009 \mathrm{kHz}$ (see column Exp. (TMC-1) in Table 2). The rotational constant is slightly below that of $\mathrm{C}_{3} \mathrm{~S}(B=2890.4 \mathrm{MHz})$ and slightly higher than that of $\mathrm{HC}_{3} \mathrm{~S}(B=2688.4 \mathrm{MHz}$; Hirahara et al. 1994) and of $\mathrm{HC}_{3} \mathrm{P}(B=2656.4 \mathrm{MHz}$; Bizzocchi et al. 2001). In our previous discovery of $\mathrm{HC}_{3} \mathrm{O}^{+}$in this source, we performed calculations for several protonated species of abundant neutral molecules in order to search for them in our survey. $\mathrm{HC}_{3} \mathrm{~S}^{+}$was one of the best candidates for detection in TMC-1. Ab initio calculations by Thorwirth et al. (2020) indicate a rotational constant for this species of $2734.5 \mathrm{MHz}$, which is very close to the value we have derived for the new molecule. We made additional calculations at a different level of theory (see Sect. 4) and found that the best prediction for $B$ and $D$ of $\mathrm{HC}_{3} \mathrm{~S}^{+}$(see Table 2) match those of the new species perfectly. Laboratory measurements (see Sect. 5) confirm that the four observed lines belong to $\mathrm{HC}_{3} \mathrm{~S}^{+}$.

From the line parameters in Table 1, adopting a dipole moment of $1.73 \mathrm{D}$ (see Sect. 4), and assuming a uniform source with a radius of $40^{\prime \prime}$ (Fossé et al. 2001), we derive a column density of $N\left(\mathrm{HC}_{3} \mathrm{~S}^{+}\right)=(2.0 \pm 0.4) \times 10^{11} \mathrm{~cm}^{-2}$ and a rotational temperature of $10 \pm 2 \mathrm{~K}$. This value is compatible with the upper limit of $3 \times 10^{11} \mathrm{~cm}^{-2}$ obtained by Cernicharo et al. (2020a) from a search based on ab initio calculations. The sensitivity

\footnotetext{
2 http://www.iram.fr/IRAMFR/GILDAS
}

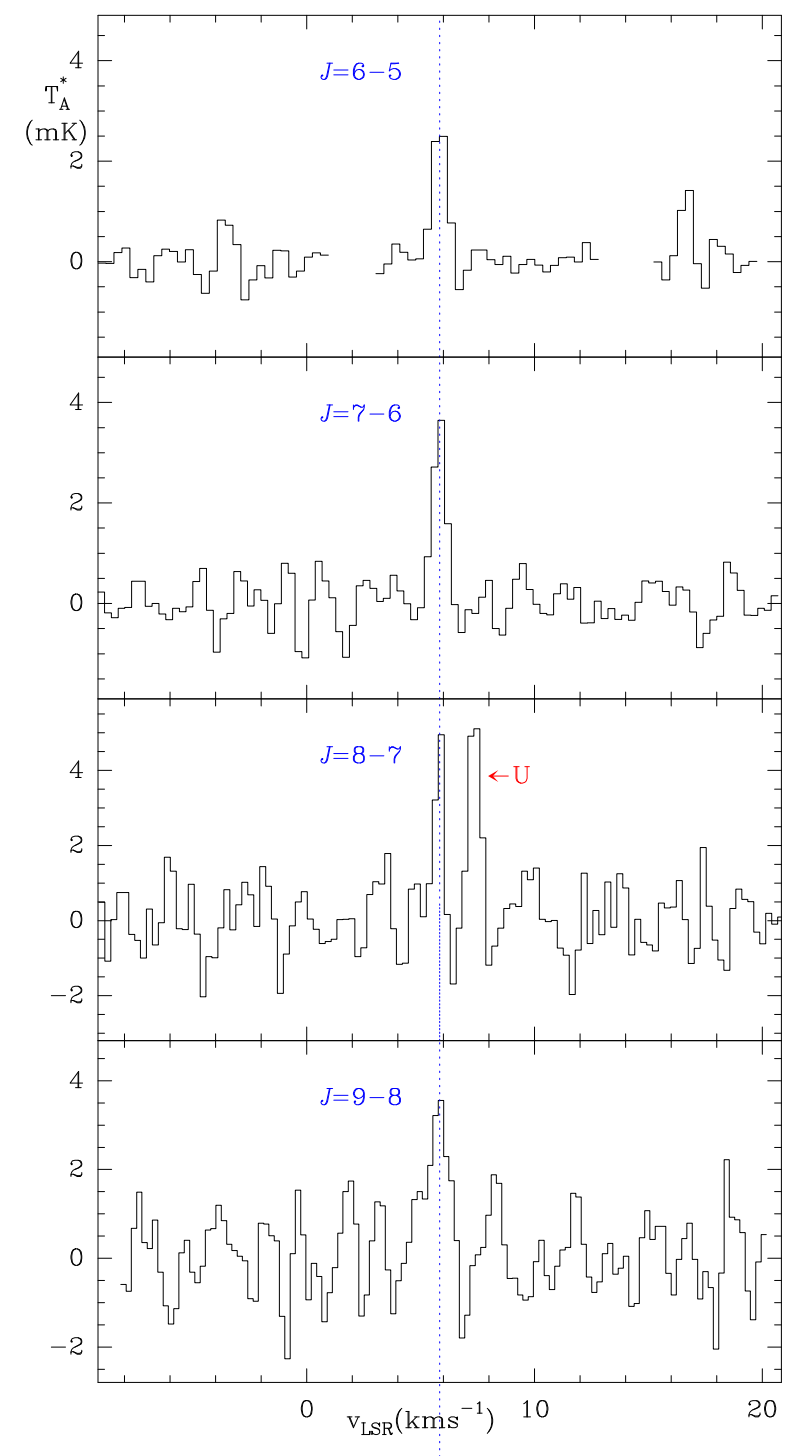

Fig. 1. Observed lines of the new molecule found in the $31-50 \mathrm{GHz}$ domain towards TMC-1. The abscissa corresponds to the local standard of rest velocity in $\mathrm{km} \mathrm{s}^{-1}$. Frequencies and intensities for the observed lines are given in Table 1 . The ordinate is the antenna temperature corrected for atmospheric and telescope losses in $\mathrm{mK}$. Spectral resolution is $38.15 \mathrm{kHz}$. Blanked channels in the top panel correspond to negative features produced in the folding of the frequency-switching observations.

improvement added by the new data has permitted its detection in TMC-1.

Protonated $\mathrm{C}_{2} \mathrm{~S}$ was predicted to be abundant in cold dense clouds by Agúndez et al. (2015) based on its high proton affinity and large abundance of $\mathrm{C}_{2} \mathrm{~S}$. We therefore carried out ab initio calculations for $\mathrm{HC}_{2} \mathrm{~S}^{+}$, which has a ${ }^{3} \Sigma$ ground electronic state (see also Puzzarini 2008), to search for the lines $N_{N} \rightarrow$ $N-1_{N-1}(J=N)$, which could be in good harmonic relation. Our estimates for $B$ and $D$ are $6048 \mathrm{MHz}$ and $0.12 \mathrm{kHz}$, respectively. These lines should exhibit a weak hyperfine splitting of $\sim 0.8 \mathrm{MHz}$. Two of these lines $\left(3_{3}-2_{2}\right.$ and $\left.4_{4}-3_{3}\right)$ fall within our $Q$-band survey. However, we have not found two harmonically related features $(3: 4)$ that could be attributed to them. The explored ranges for the $3_{3}-2_{2}$ and $4_{4}-3_{3}$ transitions are $\pm 40 \mathrm{MHz}$ around 36288 and $48384 \mathrm{MHz}$, respectively. The sensitivity of our data in these ranges is $\sim 0.5$ and $\sim 1 \mathrm{mK}$, respectively. The 
Table 1. Observed line parameters for $\mathrm{HC}_{3} \mathrm{~S}^{+}$in the laboratory and in TMC-1.

\begin{tabular}{cccccc}
\hline \hline$J_{\mathrm{u}}-J_{\mathrm{l}}$ & $\begin{array}{c}v_{\mathrm{obs}}{ }^{(a)} \\
(\mathrm{MHz})\end{array}$ & $\begin{array}{c}v_{o}-v_{c}{ }^{(b)} \\
(\mathrm{kHz})\end{array}$ & $\begin{array}{c}\int T_{\mathrm{A}}^{*} \mathrm{~d} v^{(c)} \\
\left(\mathrm{mK} \mathrm{km} \mathrm{s}^{-1}\right)\end{array}$ & $\begin{array}{c}\Delta v^{(d)} \\
\left(\mathrm{km} \mathrm{s}^{-1}\right)\end{array}$ & $\begin{array}{c}T_{\mathrm{A}}^{*} \\
(\mathrm{mK})\end{array}$ \\
\hline $3-2^{(e)}$ & 16412.7583 & -1.8 & $\ldots$ & $\ldots$ & $\ldots$ \\
$4-3^{(e)}$ & 21883.6632 & 2.4 & $\ldots$ & $\ldots$ & $\ldots$ \\
$5-4^{(e)}$ & 27354.5443 & -0.8 & $\ldots$ & $\ldots$ & $\ldots$ \\
$6-5$ & 32825.4090 & 0.0 & $1.71 \pm 0.40$ & $0.61 \pm 0.12$ & $2.6 \pm 0.4$ \\
$7-6$ & 38296.2400 & -7.5 & $1.74 \pm 0.30$ & $0.52 \pm 0.09$ & $3.1 \pm 0.4$ \\
$8-7$ & 43767.0670 & 9.5 & $2.05 \pm 0.30$ & $0.40 \pm 0.15$ & $3.9 \pm 0.4$ \\
$9-8$ & 49237.8310 & -3.4 & $3.20 \pm 0.95$ & $0.79 \pm 0.15$ & $3.6 \pm 0.9$ \\
\hline
\end{tabular}

Notes. ${ }^{(a)}$ Observed frequencies in the laboratory (below $30 \mathrm{GHz}$ ) and towards TMC-1 (above $30 \mathrm{GHz}$ ). For this source we adopted a $v_{\mathrm{LSR}}$ of $5.83 \mathrm{~km} \mathrm{~s}^{-1}$ (Cernicharo et al. 2020a,b,c). The frequency uncertainty is 3 and $10 \mathrm{kHz}$ for the laboratory and astronomical lines, respectively. ${ }^{(b)}$ Observed minus calculated frequencies in $\mathrm{kHz}$. The calculated frequencies are obtained from the merged fit to the astronomical and laboratory measurements (see Sect. 5). ${ }^{(c)}$ Integrated line intensity in $\mathrm{mK} \mathrm{km} \mathrm{s}{ }^{-1} \cdot{ }^{(d)}$ Line width at half intensity derived by fitting a Gaussian function to the observed line profile (in $\mathrm{km} \mathrm{s}^{-1}$ ). ${ }^{(e)}$ Line observed in the laboratory (see Sect. 5).

other components of each triplet $(J=N \pm 1)$ will show a more complex pattern due to the spin-spin and spin-rotation interaction. We adopted the $\lambda$ parameter from $\mathrm{C}_{2} \mathrm{~S}$, which is isoelectronic with $\mathrm{HC}_{2} \mathrm{~S}^{+}$, but the predictions have a large uncertainty and therefore a radioastronomical search is not straightforward for these transitions. Using the dipole moment derived in our ab initio calculations $(2.67 \mathrm{D})$, and assuming a rotational temperature similar to that of CCS $(5 \mathrm{~K})$, we derive a $3 \sigma$ upper limit for its column density $\leq 9 \times 10^{11} \mathrm{~cm}^{-2}$. For $T_{\mathrm{r}}=10 \mathrm{~K}$, the $3 \sigma$ upper limit is $3 \times 10^{11} \mathrm{~cm}^{-2}$. Nevertheless, the lines searched here are not best suited for a detection of $\mathrm{HC}_{2} \mathrm{~S}^{+}$as they are expected to be much higher in energy that those of the $J=N+1$ series, which, as mentioned above, require a good estimate of the spinspin interaction constant, $\lambda$, to obtain reliable frequencies with which to carry out a search for this molecule.

We also derived column densities for $S$-bearing molecules related to $\mathrm{HC}_{3} \mathrm{~S}^{+}$using our $Q$-band line survey of TMC- 1 . The line parameters are given in Table A.1. For CS and $\mathrm{HCS}^{+}$we only observed the $J=1-0$ transition and therefore we adopted a rotational temperature of $10 \mathrm{~K}$. A similar approach was taken by Vastel et al. (2018) in their study of L1544. The column density of CS, whose $J=1-0$ line has a significant optical depth, was derived from that of $\mathrm{C}^{34} \mathrm{~S}$ adopting the ${ }^{32} \mathrm{~S} /{ }^{34} \mathrm{~S}$ abundance ratio of $25 \pm 5$ determined from $\mathrm{C}_{3} \mathrm{~S}$ and $\mathrm{C}_{3}{ }^{34} \mathrm{~S}$. The derived column densities for all species studied in this paper are given in Table 3. The derived column densities for CCS and $\mathrm{C}_{3} \mathrm{~S}$ are in good agreement with those derived by Saito et al. (1987) and Yamamoto et al. (1987). A detailed analysis of the effect of the assumed rotational temperature on diatomic or linear polyatomic molecules is provided in Appendix A.

\section{Quantum chemical calculations for $\mathrm{HC}_{3} \mathrm{~S}^{+}$}

In order to obtain precise geometries and spectroscopic molecular parameters that help in the assignment of the observed lines we carried out high-level ab initio calculations for $\mathrm{HC}_{3} \mathrm{~S}^{+}$ using the Molpro 2018.1 (Werner et al. 2018) and Gaussian09 (Frisch et al. 2013) program packages. We followed the same strategy used previously for $\mathrm{HC}_{3} \mathrm{O}^{+}$(Cernicharo et al. 2020a), whereby we scaled our calculations with a molecular system that is isoelectronic to the target molecule. In the present case, we chose $\mathrm{HC}_{3} \mathrm{P}$ (see also Thorwirth et al. 2020), whose rotational parameters have been experimentally determined by Bizzocchi et al. (2001), as a reference system to scale the $\mathrm{HC}_{3} \mathrm{~S}^{+}$calculations. The geometry optimisation calculations were carried out at CCSD(T)-F12/cc-pCVTZ-F12 level of theory (Raghavachari et al. 1989; Adler et al. 2007; Knizia et al. 2009; Hill et al. 2010; Hill \& Peterson 2010), which has proven to be a suitable method with which to accurately reproduce the molecular geometry of analogue molecules (Cernicharo et al. 2019, 2020a). We first calculated $B_{e}$ for $\mathrm{HC}_{3} \mathrm{P}$ and then computed $B_{0}$ using the zero-point vibrational contribution calculated at MP2/cc-pVTZ level of theory. The agreement with the experimental value is very good, with a relative error of $0.02 \%$ (see Table 2). The $B_{0}$ value for $\mathrm{HC}_{3} \mathrm{~S}^{+}$was calculated using the $B_{e}$ value and the zero-point vibrational correction, obtained at CCSD(T)-F12/cc-pCVTZ-F12 and MP2/cc-pVTZ levels of theory, respectively. This value was then corrected using a scaling factor obtained from the ratio between the experimental and theoretical values derived for $\mathrm{HC}_{3} \mathrm{P}$. The final value of $B_{0}$ obtained for $\mathrm{HC}_{3} \mathrm{~S}^{+}$agrees very well with that obtained from observations and in the laboratory, with a relative error of around $0.01 \%$. In addition, the centrifugal distortion value obtained using the same procedure at MP2/cc-pVTZ level of theory is compatible with that obtained from the fit of the lines. The computed dipole moment is $1.73 \mathrm{D}$. The results of our calculations are in agreement with those obtained by Thorwirth et al. (2020), which were made using the same procedure but at a different level of theory.

\section{Laboratory detection of $\mathrm{HC}_{3} \mathrm{~S}^{+}$}

The rotational spectrum of $\mathrm{HC}_{3} \mathrm{~S}^{+}$was measured using a BalleFlygare-type Fourier transform microwave (FTMW) spectrometer combined with a pulsed discharge nozzle (Endo et al. 1994; Cabezas et al. 2016), which was previously used to characterise other highly reactive molecules. The transient species, $\mathrm{HC}_{3} \mathrm{~S}^{+}$, was produced in a supersonic expansion by a pulsed electric discharge of a gas mixture of $\mathrm{C}_{2} \mathrm{H}_{2}(0.2 \%), \mathrm{H}_{2}(5 \%)$, and $\mathrm{CS}_{2}$ $(0.2 \%)$ diluted in $\mathrm{Ne}$ and applying a voltage of $1100 \mathrm{~V}$ through the throat of the nozzle source. The rotational constants derived from the astronomical observations were used to predict the frequencies of the rotational transitions $J=3-2,4-3$, and 5-4 of $\mathrm{HC}_{3} \mathrm{~S}^{+}$. A scan of $\pm 2 \mathrm{MHz}$ was achieved around these frequencies and three lines were observed at 16412.7583, 21883.6632, and $27354.5443 \mathrm{MHz}$ with an uncertainty of $3 \mathrm{kHz}$ (see Fig. 2 and Table 1), just $1-5 \mathrm{kHz}$ away from the predicted frequencies using the derived rotational and distortion constants from the TMC-1 observations. The following experimental results confirm that these lines belong to a transient species: (i) they disappear in the absence of electric discharge, and (ii) the lines disappear when $\mathrm{CS}_{2}$ is removed from the gas mixture. No more lines at lower or higher frequencies $(J=2-1$ and 6-5) could be observed due to the spectrum weakness and the poorer performance of the spectrometer at those frequencies.

The fitted rotational and distortion constants derived from the laboratory data alone are given in column Exp. (labo) of Table 2. A merged fit to the laboratory and astronomical frequencies provides a rotational constant $B=2735.46311 \pm 0.00023 \mathrm{MHz}$ and a distortion constant $D=0.1720 \pm 0.0029 \mathrm{kHz}$. The standard deviation of the fit is $5.8 \mathrm{kHz}$ and the correlation coefficient between $B$ and $D$ is 0.82 . These are the recommended constants to predict the rotational spectrum of $\mathrm{HC}_{3} \mathrm{~S}^{+}$. The predicted frequencies, Einstein coefficients, upper energy levels, and line strengths for rotational transitions with $J \leq 30$ are given in Table B.1. The observed minus calculated frequencies from this merged fit are given in Table 1. 
Table 2. Theoretical and experimental values for the spectroscopic parameters of $\mathrm{HC}_{3} \mathrm{~S}^{+}$(all in $\mathrm{MHz}$ ).

\begin{tabular}{|c|c|c|c|c|c|c|c|}
\hline \multirow[b]{2}{*}{ Parameter } & \multicolumn{2}{|r|}{$\mathrm{HC}_{3} \mathrm{P}$} & \multicolumn{5}{|c|}{$\mathrm{HC}_{3} \mathrm{~S}^{+}$} \\
\hline & Calc. ${ }^{(a)}$ & Exp. ${ }^{(b)}$ & Calc. ${ }^{(a)}$ & Scaled ${ }^{(c)}$ & Exp. $\left(\right.$ TMC-1) ${ }^{(d)}$ & Exp. $(\text { Labo })^{(d)}$ & Exp. (Merged) ${ }^{(d)}$ \\
\hline $\begin{array}{l}B_{e} \\
\text { Vib-Rot. Corr. }\end{array}$ & $\begin{array}{c}2658.238 \\
2.430\end{array}$ & & $\begin{array}{c}2736.349 \\
1.801\end{array}$ & & & & \\
\hline & 2655.808 & $2656.393295(52)$ & 2734.548 & 2735.150 & $2735.4630(12)$ & $2735.46310(70)$ & $2735.46311(23)$ \\
\hline$D \times 10^{3}$ & 0.171 & $0.1810132(39)$ & 0.162 & 0.171 & $0.171(9)$ & $0.172(17)$ & $0.1720(29)$ \\
\hline
\end{tabular}

Notes. Numbers in parentheses are $1 \sigma$ uncertainties in units of the last digits. ${ }^{(a)} B$ and $D$ derived from ab initio calculations in this work. ${ }^{(b)}$ Bizzocchi et al. (2001). ${ }^{(c)}$ This work; scaled by the ratio Exp/Calc. of the corresponding parameter for $\mathrm{HC}_{3} \mathrm{P}$ species. ${ }^{(d)}$ Experimental rotational and distortion constants derived in this work.

Table 3. Column densities derived for $S$-bearing species in TMC-1.

\begin{tabular}{lcc}
\hline \hline Molecule & $N\left(\mathrm{~cm}^{-2}\right)$ & $T_{\text {rot }}(\mathrm{K})$ \\
\hline $\mathrm{CS}^{(a)}$ & $(3.50 \pm 0.40) \times 10^{14}$ & $10.0^{(b)}$ \\
$\mathrm{C}^{34} \mathrm{~S}$ & $(1.45 \pm 0.10) \times 10^{13}$ & $10.0^{(b)}$ \\
${ }^{13} \mathrm{C}^{34} \mathrm{~S}$ & $(1.45 \pm 0.20) \times 10^{11}$ & $10.0^{(b)}$ \\
$\mathrm{HCS}^{+}$ & $(1.00 \pm 0.10) \times 10^{13}$ & $10.0^{(b)}$ \\
$\mathrm{HC}^{34} \mathrm{~S}^{+}$ & $(7.10 \pm 0.70) \times 10^{11}$ & $10.0^{(b)}$ \\
$\mathrm{CCS}^{34}$ & $(5.50 \pm 0.65) \times 10^{13}$ & $5.1 \pm 0.2$ \\
$\mathrm{CC}^{34} \mathrm{~S}$ & $(5.00 \pm 0.50) \times 10^{12}$ & $3.6 \pm 0.4$ \\
$\mathrm{CCCS}$ & $(1.30 \pm 0.13) \times 10^{13}$ & $5.8 \pm 0.2$ \\
$\mathrm{CCC}^{34} \mathrm{~S}$ & $(5.30 \pm 0.50) \times 10^{11}$ & $6.7 \pm 0.2$ \\
$\mathrm{HC}_{3} \mathrm{~S}^{+}$ & $(2.00 \pm 0.40) \times 10^{11}$ & $10.0 \pm 2.0$ \\
$\mathrm{HCCS}^{+(c)}$ & $\leq 9.00 \times 10^{11}$ & $10.0^{(b)}$ \\
\hline
\end{tabular}

Notes. ${ }^{(a)}$ Derived from $\mathrm{C}^{34} \mathrm{~S}$ and the $\mathrm{C}_{3} \mathrm{~S}_{\mathrm{C}} \mathrm{C}_{3}{ }^{34} \mathrm{~S}$ abundance ratio. ${ }^{(b)}$ Rotational temperature has been fixed. See Appendix A. ${ }^{(c)} 3 \sigma$ upper limit.

\section{Chemical model}

From Table 3 we derive the following neutral-to-protonated column density ratios: $\mathrm{CS} / \mathrm{HCS}^{+}=35 \pm 6, \mathrm{C}_{2} \mathrm{~S} / \mathrm{HC}_{2} \mathrm{~S}^{+} \geq 60$, and $\mathrm{C}_{3} \mathrm{~S} / \mathrm{HC}_{3} \mathrm{~S}^{+}=65 \pm 20$. In order to interpret these ratios we carried out chemical model calculations similar to those presented by Agúndez et al. (2015), who discussed the chemistry of protonated molecules in cold dense clouds. We have adopted the KIDA kida. uva. 2014 chemical network (Wakelam et al. 2015). The abundances of sulfur-bearing molecules are strongly dependent on the depletion of sulfur, which is still a matter of debate (Vidal et al. 2017; Vastel et al. 2018). Here we have adopted the so-called set of low-metal elemental abundances (see Agúndez \& Wakelam 2013), in which $S / H=8 \times 10^{-8}$. The resulting fractional abundances and abundance ratios are shown in Fig. 3 as a function of time. If we focus on the so-called early time $\left(10^{5}-10^{6} \mathrm{yr}\right)$, at which calculated abundances agree better with observations (e.g. Agúndez \& Wakelam 2013), we see that the chemical model reproduces the observed fractional abundances of neutral and protonated molecules reasonably well, at the exception of $\mathrm{C}_{2} \mathrm{~S}$ and $\mathrm{HCS}^{+}$, which translates to good agreement between calculated and observed $[\mathrm{M}] /\left[\mathrm{MH}^{+}\right]$ratios for $\mathrm{M}=\mathrm{C}_{3} \mathrm{~S}$ but not for $\mathrm{M}=\mathrm{CS}$ and $\mathrm{C}_{2} \mathrm{~S}$.

As discussed by Agúndez et al. (2015), in a simplified chemical scheme, a protonated molecule is formed through proton transfer from a proton donor (typically $\mathrm{HCO}^{+}, \mathrm{H}_{3} \mathrm{O}^{+}$, and $\mathrm{H}_{3}^{+}$) and destroyed by dissociative recombination with electrons. In that case, the neutral-to-protonated abundance ratio at steady state is simply given by the ratio of rate constants of the reaction

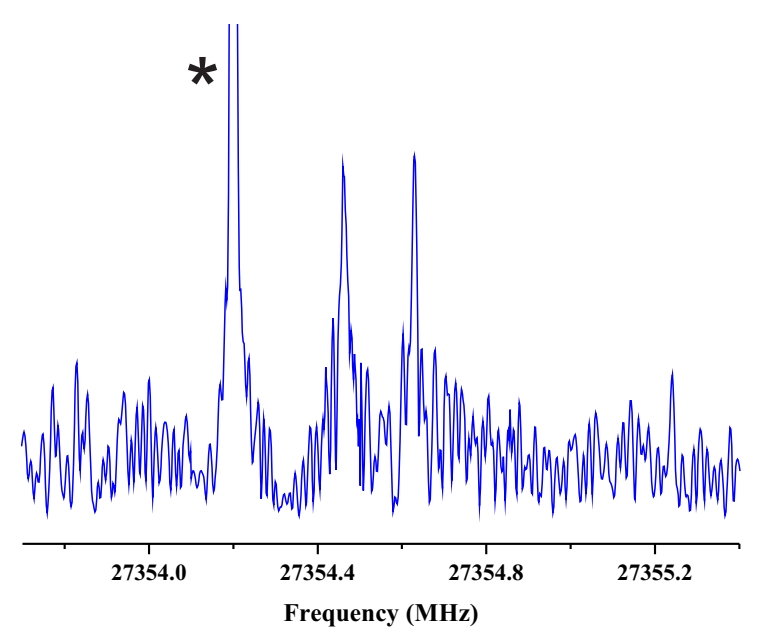

Fig. 2. FTMW spectra of $\mathrm{HC}_{3} \mathrm{~S}^{+}$showing the $J=5-4$ rotational transition at $27.3 \mathrm{GHz}$. The spectrum was achieved by 10000 shots of accumulation at a repetition rate of $10 \mathrm{~Hz}$. The coaxial arrangement of the adiabatic expansion and the resonator axis produces an instrumental Doppler doubling. The resonance frequency is calculated as the average of the two Doppler components. The feature marked with an asterisk is an artifact.

of dissociative recombination and of proton transfer multiplied by the abundance ratio between electrons and the proton donor. This simple scheme should hold unless there are important alternative ion-molecule reactions of formation of the ion other than proton transfer to the neutral.

For $\mathrm{HC}_{3} \mathrm{~S}^{+}$the chemical model indicates that the main formation reactions are proton transfer to $\mathrm{C}_{3} \mathrm{~S}$ from $\mathrm{HCO}^{+}$and $\mathrm{H}_{3} \mathrm{O}^{+}$, although the reactions $\mathrm{S}^{+}+\mathrm{C}_{3} \mathrm{H}_{2}$ (cyclic or linear) and $\mathrm{S}+\mathrm{C}_{3} \mathrm{H}_{3}^{+}$(cyclic or linear) are also efficient at the same level. The reactions of $\mathrm{S}^{+}$with both cyclic and linear $\mathrm{C}_{3} \mathrm{H}_{2}$ have been calculated to be barrierless when leading to $\mathrm{HC}_{3} \mathrm{~S}^{+}$ (Redondo et al. 1999). However, the cyclic isomer of $\mathrm{C}_{3} \mathrm{H}_{2}$ is 28 times more abundant than the linear one in TMC-1 (Fossé et al. 2001), and thus the reaction $\mathrm{S}^{+}+\mathrm{c}_{-} \mathrm{C}_{3} \mathrm{H}_{2}$ will contribute significantly more than $\mathrm{S}^{+}+\mathrm{H}_{2} \mathrm{C}_{3}$ to the formation of $\mathrm{HC}_{3} \mathrm{~S}^{+}$. The fact that the calculated $\mathrm{C}_{3} \mathrm{~S} / \mathrm{HC}_{3} \mathrm{~S}^{+}$ratio is in agreement with the observed value supports the hypothesis that $\mathrm{HC}_{3} \mathrm{~S}^{+}$is formed by the aforementioned reactions.

In the case of $\mathrm{HCS}^{+}$, the chemical model underestimates its formation, which is carried out by proton transfer to $\mathrm{CS}$ from $\mathrm{H}_{3} \mathrm{O}^{+}$and $\mathrm{HCO}^{+}$and by the reaction between $\mathrm{CS}^{+}$and $\mathrm{H}_{2}$. Including grain-surface chemical reactions, Vidal et al. (2017) calculate a CS$/ \mathrm{HCS}^{+}$ratio in better agreement with observations, especially at late times. The reason for the low abundance 

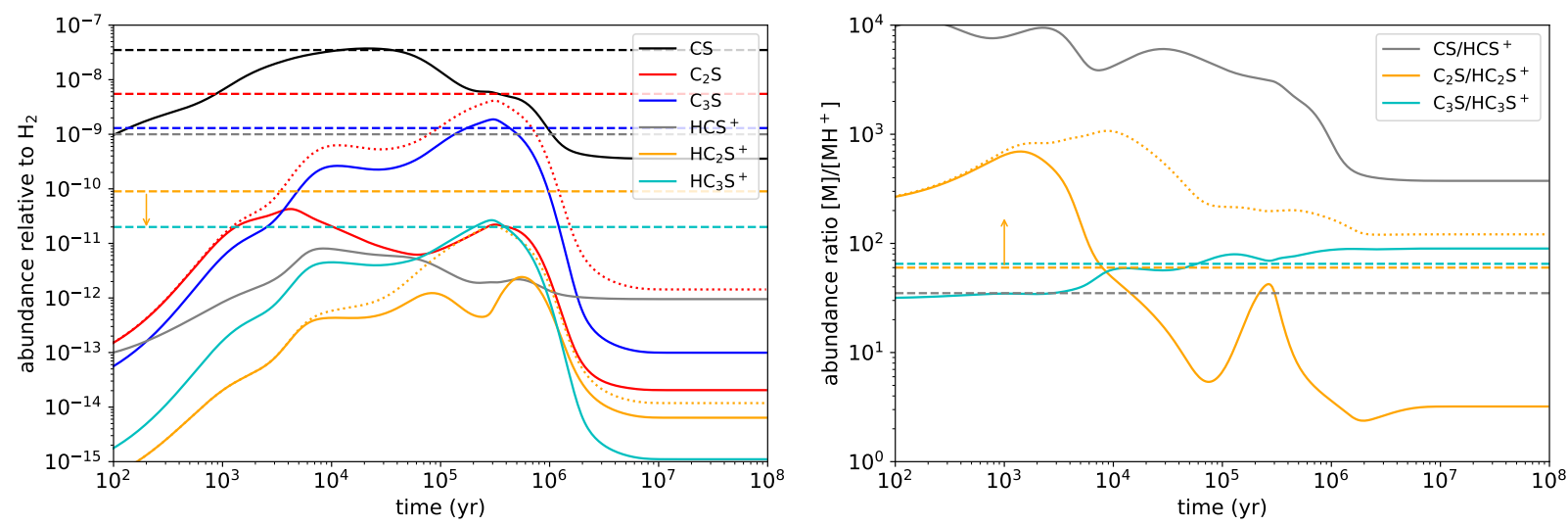

Fig. 3. Calculated abundances (left) and abundance ratios (right) as a function of time for a cold dense cloud. Observed values in TMC-1 (see Table 3) are indicated by horizontal dashed lines. The observed $\mathrm{HC}_{2} \mathrm{~S}^{+}$abundance and $\mathrm{C}_{2} \mathrm{~S} / \mathrm{HC}_{2} \mathrm{~S}^{+}$ratio are an upper and a lower limit, respectively, and are indicated by vertical arrows. Dotted lines represent the calculated abundances of $\mathrm{C}_{2} \mathrm{~S}$ and $\mathrm{HC}_{2} \mathrm{~S}^{+}$(left panel) and its ratio (right panel) when the reaction $\mathrm{O}+\mathrm{C}_{2} \mathrm{~S}$ is neglected.

of $\mathrm{HCS}^{+}$in our gas-phase model may be related to the underproduction of $\mathrm{H}_{2} \mathrm{~S}$ (see e.g. Agúndez \& Wakelam 2013), which is enhanced when including grain-surface chemistry, and which can increase the abundance of $\mathrm{HCS}^{+}$through reactions like $\mathrm{H}_{2} \mathrm{~S}$ $+\mathrm{C}^{+}$and $\mathrm{H}_{2} \mathrm{~S}^{+}+\mathrm{C}$.

While the calculated abundance of $\mathrm{HC}_{2} \mathrm{~S}^{+}$is in agreement with the observed upper limit, the calculated $\mathrm{C}_{2} \mathrm{~S} / \mathrm{HC}_{2} \mathrm{~S}^{+}$ratio is too low because the chemical model severely underestimates the abundance of $\mathrm{C}_{2} \mathrm{~S}$. This is because the chemical network kida.uva.2014 includes an efficient destruction channel for $\mathrm{C}_{2} \mathrm{~S}$ through reaction with $\mathrm{O}$ atoms, based on estimations by Loison et al. (2012). If this reaction is neglected, the calculated abundance of $\mathrm{C}_{2} \mathrm{~S}$ is shifted up and becomes very close to the observed value, while the abundance of $\mathrm{HC}_{2} \mathrm{~S}^{+}$also increases, remaining consistent with the observed upper limit (see dotted lines in Fig. 3). Accurate calculations of the rate constant of the $\mathrm{O}+\mathrm{C}_{2} \mathrm{~S}$ reaction at low temperatures are needed to shed light on the chemistry of $\mathrm{C}_{2} \mathrm{~S}$ in cold dense clouds.

\section{Conclusions}

We report the first identification in space of protonated $C_{3} S$. Four harmonically related lines observed toward TMC-1 using the Yebes $40 \mathrm{~m}$ radiotelescope have been unambiguously assigned to this ion thanks to accurate ab initio quantum chemical calculations and laboratory measurements of the rotational spectrum of this species. The derived $\mathrm{C}_{3} \mathrm{~S} / \mathrm{HC}_{3} \mathrm{~S}^{+}$ratio of $55 \pm 20$ is well reproduced by a gas-phase chemical model in which $\mathrm{HC}_{3} \mathrm{~S}^{+}$ is mostly formed through protonation of $\mathrm{C}_{3} \mathrm{~S}$ and the reactions $\mathrm{S}^{+}+\mathrm{C}_{3} \mathrm{H}_{2}$ and $\mathrm{S}+\mathrm{C}_{3} \mathrm{H}_{3}^{+}$.

Acknowledgements. We thank Ministerio de Ciencia e Innovación of Spain (MICIU) for funding support through projects AYA2016-75066-C21-P, PID2019-106110GB-I00, PID2019-107115GB-C21 / AEI / 10.13039/ 501100011033, and PID2019-106235GB-I00. We also thank ERC for funding through grant ERC-2013-Syg-610256-NANOCOSMOS. M.A. thanks MICIU for grant RyC-2014-16277. Y.E. thanks Ministry of Science and Technology of Taiwan through grant MOST108-2113-M-009-25.

\section{References}

Adler, T. B., Knizia, G., \& Werner, H.-J. 2007, J. Chem. Phys., 127, 221106 Agúndez, M., \& Wakelam, V. 2013, Chem. Rev., 113, 8710

Agúndez, M., Cernicharo, J., de Vicente, P., et al. 2015, A\&A, 579, L10
Bizzocchi, L., Thorwirth, S., \& Müller, H. S. P. 2001, J. Mol. Spectrosc., 205, 110

Cabezas, C., Guillemin, J.-C., \& Endo, Y. 2016, J. Chem. Phys., 145, 184304

Cernicharo, J. 1985, Internal IRAM Report (Granada: IRAM)

Cernicharo, J. 2012, in ECLA 2011: Proc. of the European Conference on Laboratory Astrophysics, eds. C. Stehl, C. Joblin, \& L. d'Hendecourt (Cambridge: Cambridge Univ. Press), EAS Publ. Ser., 251, https:// nanocosmos.iff.csic.es/?page_id=1619

Cernicharo, J., Guélin, M., Hein, H., \& Kahane, C. 1987, A\&A, 181, L9

Cernicharo, J., Cabezas, C., Pardo, J. R., et al. 2019, A\&A, 630, L2

Cernicharo, J., Marcelino, N., Agúndez, M., et al. 2020a, A\&A, 642, L17

Cernicharo, J., Marcelino, N., Pardo, J. R., et al. 2020b, A\&A, 641, L9

Cernicharo, J., Marcelino, N., Agúndez, M., et al. 2020c, A\&A, 642, L8

Denis-Alpizar, O., Stoecklin, T., Guilloteau, S., \& Dutrey, A. 2018, MNRAS, 478, 1811

Endo, Y., Kohguchi, H., \& Ohshima, Y. 1994, Faraday Discuss., 97, 341

Fossé, D., Cernicharo, J., Gerin, M., \& Cox, P. 2001, ApJ, 552, 168

Frisch, M. J., Trucks, G. W., Schlegel, H. B., et al. 2013, Gaussian 09, Revision D.01

Goldsmith, P. F., \& Langer, W. D. 1999, ApJ, 517, 209

Gordy, W., \& Cook, R. L. 1984, Microwave Molecular Spectra, Chapter V (New York: Wiley)

Hill, J. G., \& Peterson, K. A. 2010, Phys. Chem. Chem. Phys., 12, 10460

Hill, J. G., Mazumder, S., \& Peterson, K. A. 2010, J. Chem. Phys., 132, 054108

Hirahara, Y., Ohshima, Y., \& Endo, Y. 1994, J. Chem. Phys., 101, 7342

Hunter, E. P. L., \& Lias, S. G. 1998, J. Phys. Chem. Ref. Data, 27, 413

Kaifu, N., Ohishi, M., Kawaguchi, K., et al. 2004, PASJ, 56, 69

Kawaguchi, K., Kasai, Y., Ishikawa, S.-I., et al. 1994, ApJ, 420, L95

Knizia, G., Adler, T. B., \& Werner, H.-J. 2009, J. Chem. Phys., 130, 054104

Loison, J.-C., Halvick, P., Bergeat, A., et al. 2012, MNRAS, 421, 1476

Marcelino, N., Agúndez, M., Tercero, B., et al. 2020, A\&A, 643, L6

Müller, H. S. P., Schlöder, F., Stutzki, J., \& Winnewisser, G. 2005, J. Mol. Struct., 742,215

Pardo, J. R., Cernicharo, J., \& Serabyn, E. 2001, IEEE Trans. Antennas Propag., 49, 12

Pickett, H. M., Poynter, R. L., Cohen, E. A., et al. 1998, J. Quant. Spectrosc. Radiat. Trans., 60, 883

Puzzarini, C. 2008, Chem. Phys., 346, 45

Raghavachari, K., Trucks, G. W., Pople, J. A., \& Head-Gordon, M. 1989, Chem. Phys. Lett., 157, 479

Redondo, P., Calleja, E., Barrientos, C., \& Largo, A. 1999, J. Phys. Chem. A, 103,9125

Saito, S., Kawaguchi, K., Yamamoto, S., et al. 1987, ApJ, 317, L1156

Tercero, F., López-Pérez, J. A., Gallego, J. D., et al. 2021, A\&A, 645, A37

Thorwirth, S., Harding, M. E., Asvany, O., et al. 2020, Mol. Phys., 118, e1776409

Vastel, C., Quénard, D., Le Gal, R., et al. 2018, MNRAS, 478, 5514

Vidal, T. H. G., Loison, J.-C., Jaziri, A. Y., et al. 2017, MNRAS, 469, 435

Yamamoto, S., Saito, S., Kawaguchi, K., et al. 1987, ApJ, 317, L119

Wakelam, V., Loison, J.-C., Herbst, E., et al. 2015, ApJS, 217, 20

Werner, H. J., Knowles, P. J., Knizia, G., et al. 2018, MOLPRO, version 2018.1 


\section{Appendix A: Column densities under LTE for linear molecules}

The determination of column densities from the observed line paramaters of the rotational transitions of a molecule requires knowledge of the collisional rates of the molecular species under study. These values are not always available and rotational diagrams are used to derived rotational temperatures, $T_{\mathrm{r}}$, and column densities (Goldsmith \& Langer 1999). Often only one line has been observed and therefore an assumption has to be made as to the rotational temperature. The line parameters of the species studied in this paper are given in Table A.1.

In this section we analyse how the derived column density depends on the adopted $T_{\mathrm{r}}$ for a range of frequencies and upper energy levels. Let us assume an optically thin line $(\tau \ll 1)$ with frequency $v_{\mathrm{ul}}$ and arising from an upper level with energy $E_{\mathrm{u}}$. If the medium has uniform excitation conditions, and the molecular levels are populated under a uniform rotational temperature $T_{\mathrm{r}}$, then, the observed brightness temperature $\left(T_{\mathrm{B}}\right)$ is given by:

$T_{\mathrm{B}}(v)=\left(T_{\mathrm{r}}-T_{\mathrm{bg}}\right) \tau(v)$,

where $T_{\mathrm{bg}}$ is the temperature of the cosmic radiation background, and $\tau(v)$ the opacity of the line at frequency $v$, which is given by

$\tau(v)=\frac{c^{2} A_{\mathrm{ul}} g_{\mathrm{u}}}{8 \pi v^{2}}\left(\frac{N_{1}}{g_{1}}-\frac{N_{\mathrm{u}}}{g_{\mathrm{u}}}\right) \phi(v)$,

where $N_{\mathrm{l}}, N_{\mathrm{u}}, g_{\mathrm{l}}$, and $g_{\mathrm{u}}$ are the column densities and statistical weights of the lower and upper levels, $A_{\mathrm{ul}}$ is the Einstein coefficient of the transition, and $\phi(v)$ is the normalised line profile, which for a Gaussian line is given by

$\phi(v)=\frac{c}{v_{\mathrm{ul}} \sigma \sqrt{\pi}} e^{-\left(\frac{v-v_{\mathrm{ul}}}{\sigma} \frac{c}{v_{\mathrm{ul}}}\right)^{2}}$,

where $\sigma=\Delta v /(2 \sqrt{\ln 2})$, with $\Delta v$ being the line full width at half intensity (in $\mathrm{km} \mathrm{s}^{-1}$ ). Using the following relations in which $S_{\mathrm{ul}}$ is the line strength and $\mu$ is the permanent dipole moment of the molecule,

$A_{\mathrm{ul}}=\frac{64 \pi^{4} v_{\mathrm{ul}}^{3}}{3 h c^{3}} \frac{S_{\mathrm{ul}} \mu^{2}}{g_{\mathrm{u}}}$,

$\frac{N_{\mathrm{u}} / g_{\mathrm{u}}}{N_{\mathrm{l}} / g_{\mathrm{l}}}=e^{-h v_{\mathrm{ul}} / k_{\mathrm{B}} T_{\mathrm{r}}}$

$N_{\mathrm{u}}=\frac{N}{Q_{\mathrm{rot}}} g_{\mathrm{u}} e^{-E_{\mathrm{u}} / k_{\mathrm{B}} T_{\mathrm{r}}}$,

we derive

$\tau\left(v=v_{\mathrm{ul}}\right)=\left(\frac{8 \pi^{5 / 2} S_{\mathrm{ul}} \mu^{2}}{3 h \sigma}\right)\left(\frac{N}{Q_{\mathrm{rot}}}\right) e^{-E_{\mathrm{u}} / k_{\mathrm{B}} T_{\mathrm{r}}}\left(e^{h v_{\mathrm{ul}} / k_{\mathrm{B}} T_{\mathrm{r}}}-1\right)$,

where $N$ is the column density of the molecule, $Q_{\text {rot }}$ is the rotational partition function, $h$ is the Planck constant, and $k_{\mathrm{B}}$ is the Boltzmann constant. For linear molecules, the rotational partition function (Gordy \& Cook 1984) is given by:

$Q_{\mathrm{rot}} \simeq \frac{k_{\mathrm{B}} T_{\mathrm{r}}}{h B}$

where $B$ is the rotational constant of the molecule. This approximation is rather accurate, even for low values of $T_{\mathrm{r}}$ if $T_{\mathrm{r}} / B \gg 1$.

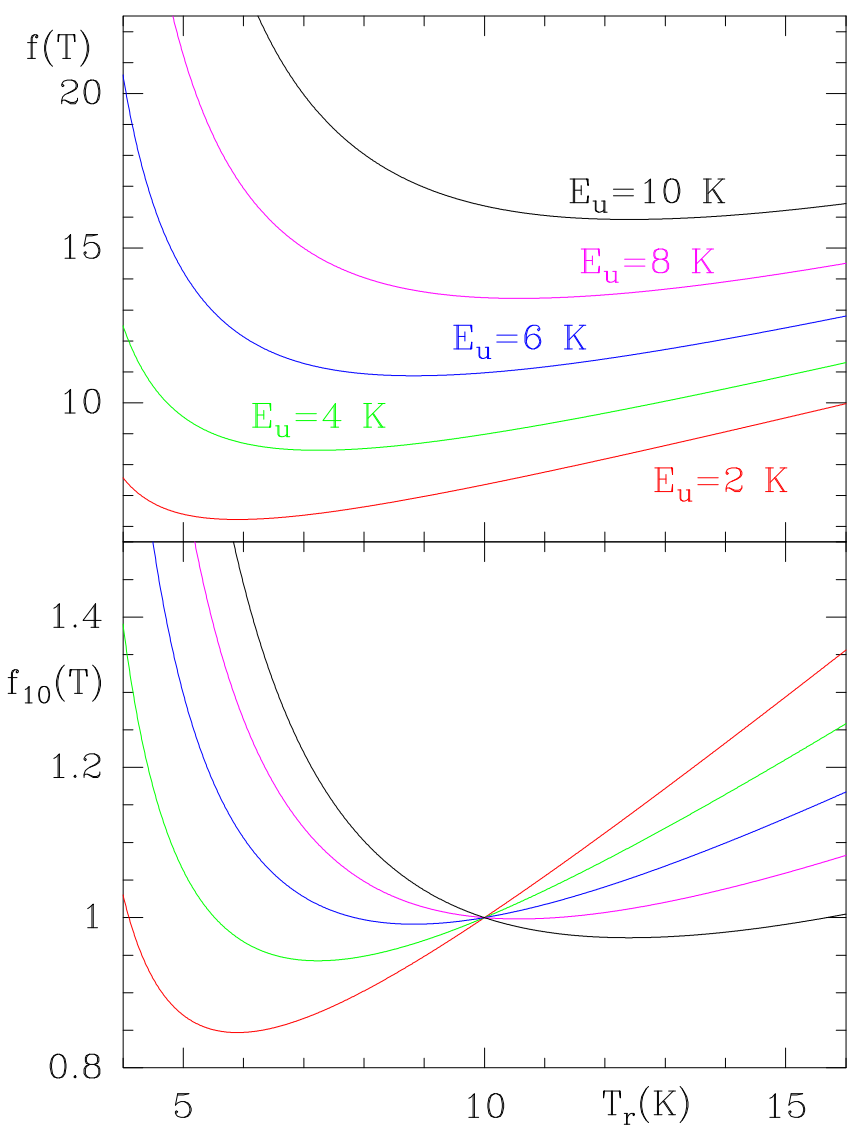

Fig. A.1. Dependency of the derived column density on the assumed rotational temperature for a line at $42.674 \mathrm{GHz}\left(\mathrm{HCS}^{+} J=1-0\right.$; upper panel). The upper energy level is varied between 2 and $10 \mathrm{~K}$. Lower panel: same function but normalised to its value for $T_{\mathrm{r}}=10 \mathrm{~K}$.

Hence, the brightness temperature at the line centre $\left(v=v_{\mathrm{ul}}\right)$ is given by

$T_{\mathrm{B}}\left(v_{u l}\right) \simeq \frac{\left(T_{r}-T_{b g}\right)}{T_{r}}\left(\frac{8 \pi^{5 / 2} S_{u l} \mu^{2} B}{3 \sigma k_{\mathrm{B}}}\right) N e^{-E_{\mathrm{u}} / k_{\mathrm{B}} T_{\mathrm{r}}}\left(e^{h v_{\mathrm{ul}} / k_{\mathrm{B}} T_{\mathrm{r}}}-1\right)$,

and the column density can be derived from the observed brightness temperature of the observed transition from the following expression:

$N \simeq C T_{\mathrm{B}}\left(v_{\mathrm{ul}}\right) \Delta v f\left(E_{\mathrm{u}}, v_{\mathrm{ul}}, T_{\mathrm{r}}\right)$,

where $C$ is a constant that depends on the molecular parameters and is given by

$$
\begin{aligned}
C & =\frac{3 k_{\mathrm{B}}}{8 \pi^{5 / 2} S_{\mathrm{ul}} \mu^{2} B \sqrt{4 \ln 2}}, \\
& =\frac{1.7774 \times 10^{14}}{S_{\mathrm{ul}} \mu^{2} B} \mathrm{~cm}^{-2} K^{-1}\left(\mathrm{kms}^{-1}\right)^{-1}
\end{aligned}
$$

in these expressions $\Delta v$ is in $\mathrm{km} \mathrm{s}^{-1}$, and corresponds to the line full width at half power intensity (see expression (A.3)), $B$ is in $\mathrm{GHz}$ and $\mu$ in D.

The function $f$ depends on $E_{\mathrm{u}}, v_{\mathrm{ul}}$, and $T_{\mathrm{r}}$ as

$f\left(E_{\mathrm{u}}, v_{\mathrm{ul}}, T_{\mathrm{r}}\right)=\frac{T_{\mathrm{r}}}{\left(T_{\mathrm{r}}-T_{\mathrm{bg}}\right)} \frac{e^{E_{\mathrm{u}} / k_{\mathrm{B}} T_{\mathrm{r}}}}{\left(e^{h v_{\mathrm{ul}} / k_{\mathrm{B}} T_{\mathrm{r}}}-1\right)}$, 
Table A.1. Observed line parameters in TMC-1 for CS, $\mathrm{HCS}^{+}, \mathrm{C}_{2} \mathrm{~S}$ and $\mathrm{C}_{3} \mathrm{~S}$.

\begin{tabular}{lcccccc}
\hline \hline Molecule & Transition & $\begin{array}{c}v_{\text {rest }}{ }^{(a)} \\
(\mathrm{MHz})\end{array}$ & $\begin{array}{c}\int T_{\mathrm{A}}^{*} \mathrm{~d} v^{(b)} \\
\left(\mathrm{Kkm} \mathrm{s}^{-1}\right)\end{array}$ & $\begin{array}{c}V_{\mathrm{LSR}}{ }^{(c)} \\
\left(\mathrm{km} \mathrm{s}^{-1}\right)\end{array}$ & $\begin{array}{c}\Delta v^{(d)} \\
\left(\mathrm{km} \mathrm{s}^{-1}\right)\end{array}$ & $\begin{array}{c}T_{\mathrm{A}}^{*}(e) \\
(\mathrm{K})\end{array}$ \\
\hline $\mathrm{CS}$ & $1-0$ & $48990.9573(1)$ & $1.0623(10)$ & $5.75(1)$ & $0.74(1)$ & $1.3451(14)$ \\
& & & $0.1031(20)$ & $6.46(1)$ & $0.72(1)$ & $0.1345(14)$ \\
$\mathrm{C}^{34} \mathrm{~S}$ & $1-0$ & $48206.9417(1)$ & $0.4025(10)$ & $5.82(1)$ & $0.65(1)$ & $0.5902(12)$ \\
${ }^{13} \mathrm{C}^{34} \mathrm{~S}$ & $1-0$ & $45463.4123(6)$ & $0.0044(10)$ & $5.72(5)$ & $0.68(9)$ & $0.0061(10)$ \\
$\mathrm{HCS}^{+}$ & $1-0$ & $42674.1954(14)$ & $0.2395(10)$ & $5.79(1)$ & $0.54(1)$ & $0.4154(7)$ \\
$\mathrm{HC}^{34} \mathrm{~S}^{+}$ & $1-0$ & $41983.0630(16)$ & $0.0138(10)$ & $5.79(1)$ & $0.58(2)$ & $0.0223(6)$ \\
$\mathrm{C}_{2} \mathrm{~S}$ & $2{ }_{3}-1_{2}$ & $33751.3699(6)$ & $1.1417(10)$ & $5.77(1)$ & $0.75(1)$ & $1.4346(4)$ \\
$\mathrm{C}_{2} \mathrm{~S}$ & $3_{3}-3_{2}$ & $38866.4200(29)$ & $0.1918(10)$ & $5.77(1)$ & $0.63(1)$ & $0.2842(5)$ \\
$\mathrm{C}_{2} \mathrm{~S}$ & $43-3_{2}$ & $43981.0190(36)$ & $0.1745(10)$ & $5.78(1)$ & $0.59(1)$ & $0.2780(7)$ \\
$\mathrm{C}_{2} \mathrm{~S}$ & $34-23$ & $45379.0275(13)$ & $1.0521(10)$ & $5.79(1)$ & $0.60(1)$ & $1.6468(7)$ \\
$\mathrm{C}_{2}{ }^{34} \mathrm{~S}$ & $23-1_{2}$ & $33111.8420(13)$ & $0.0842(10)$ & $5.79(1)$ & $0.72(1)$ & $0.1101(4)$ \\
$\mathrm{C}_{2}{ }^{34} \mathrm{~S}$ & $3_{3}-22$ & $38015.2321(23)$ & $0.0070(10)$ & $5.79(1)$ & $0.63(1)$ & $0.0100(5)$ \\
$\mathrm{C}_{2}{ }^{34} \mathrm{~S}$ & $4{ }_{3}-3_{2}$ & $42918.1822(13)$ & $0.0062(10)$ & $5.81(1)$ & $0.55(1)$ & $0.0108(6)$ \\
$\mathrm{C}_{2}{ }^{34} \mathrm{~S}$ & $3_{4}-2$ & $44497.6025(42)$ & $0.0820(10)$ & $5.82(1)$ & $0.57(1)$ & $0.1343(8)$ \\
$\mathrm{C}_{3} \mathrm{~S}$ & $6-5$ & $34684.3681(6)$ & $0.4938(10)$ & $5.80(1)$ & $0.72(1)$ & $0.6482(4)$ \\
$\mathrm{C}_{3} \mathrm{~S}$ & $7-6$ & $40465.0144(7)$ & $0.4599(10)$ & $5.81(1)$ & $0.62(1)$ & $0.6955(5)$ \\
$\mathrm{C}_{3} \mathrm{~S}$ & $8-7$ & $46245.6231(8)$ & $0.4073(10)$ & $5.82(1)$ & $0.60(1)$ & $0.6437(9)$ \\
$\mathrm{C}_{3}{ }^{34} \mathrm{~S}$ & $6-5$ & $33844.2468(9)$ & $0.0198(10)$ & $5.84(1)$ & $0.73(1)$ & $0.0255(4)$ \\
$\mathrm{C}_{3}{ }^{34} \mathrm{~S}$ & $7-6$ & $39484.8768(11)$ & $0.0200(10)$ & $5.85(1)$ & $0.64(1)$ & $0.0296(5)$ \\
$\mathrm{C}_{3}{ }^{34} \mathrm{~S}$ & $8-7$ & $45125.4708(12)$ & $0.0185(10)$ & $5.84(1)$ & $0.59(2)$ & $0.0291(9)$ \\
\hline
\end{tabular}

Notes. ${ }^{(a)}$ Predicted frequencies from the MADEX code (Cernicharo et al. 2012). Numbers in parentheses are $1 \sigma$ uncertainties in units of the last digits. ${ }^{(b)}$ Integrated line intensity in $\mathrm{K} \mathrm{km} \mathrm{s}^{-1} .{ }^{(c)}$ Line velocity with respect to the Local Standard of Rest in $\mathrm{km} \mathrm{s}^{-1}$. ${ }^{(d)}$ Linewidth at half intensity derived by fitting a Gaussian function to the observed line profile (in $\mathrm{km} \mathrm{s}^{-1}$ ). ${ }^{(e)}$ Antenna temperature in $\mathrm{K}$.

and it shows a smooth behaviour with $T_{\mathrm{r}}$ for most cases of interest in the frequency range of our survey. Figure A.1 shows the value of the function $f$ for different values of the energy of the upper level and for a frequency of $42.674 \mathrm{GHz}$ (the frequency of the $J=1-0$ transition of $\mathrm{HCS}^{+}$). The case $E_{\mathrm{u}}=2 \mathrm{~K}$ corresponds, specifically, to the $J=1-0$ transition of $\mathrm{HCS}^{+}$. For this particular case the value of $f$ for $T_{\mathrm{r}}=5 \mathrm{~K}$ is 6.59 while for $T_{\mathrm{r}}=10 \mathrm{~K}$ it is 7.56 . Hence, assuming $T_{\mathrm{r}}=10 \mathrm{~K}$ represents a change in the column density of $+15 \%$ with respect the case with $T_{r}=5 \mathrm{~K}$. The bottom panel of Fig. A. 1 shows the function $f$ normalised to its value at $T_{\mathrm{r}}=10 \mathrm{~K}$. Hence, the different plots, which correspond to different energies of the upper level (always for a frequency of $42.674 \mathrm{GHz}$ ), show the relative error on the estimated column density assuming $T_{\mathrm{r}}=10 \mathrm{~K}$ with respect other values of the rotational temperature.

For most molecules detected in the survey we observe transitions with low $J_{\mathrm{u}}$ and therefore with moderate upper energy levels. The error on the derived column density from the observed line parameters of just one transition of a linear molecule is below $20 \%$ for $E_{\mathrm{u}} \leq 6 \mathrm{~K}$ and for $T_{\mathrm{r}}$ between 5 and $10 \mathrm{~K}$. This applies particularly to $\mathrm{CS}$ and $\mathrm{HCS}^{+}$and other linear species in our survey. For transitions involving levels with $E_{\mathrm{u}} \gg T_{\mathrm{r}}$ the error on the estimated column density can be considerably larger. From Fig. A.1 we can see that a change from $T_{\mathrm{r}}=6$ to $10 \mathrm{~K}$ introduces an error of $\sim 1.5$ for a transition with $E_{\mathrm{u}}=10 \mathrm{~K}$. The error can reach a factor three if $E_{\mathrm{u}}=15 \mathrm{~K}$. For these cases a realistic estimation of the rotational temperature is needed based on the observation of several rotational transitions of the molecule under study.

An additional source of uncertainty in the column density when only one line is observed is the assumption of local thermodynamical equilibrium (LTE) under a uniform rotational temperature for all rotational levels. For the physical conditions of TMC-1, $T_{K}=10 \mathrm{~K}$ and $\mathrm{n}\left(\mathrm{H}_{2}\right)=4 \times 10^{4} \mathrm{~cm}^{-3}$ (Cernicharo et al. 1987; Fossé et al. 2001), the $J=1-0$ line of most molecules will have an excitation temperature close to the kinetic one. However, the excitation of rotational levels with higher values of $J$ will be certainly below the kinetic temperature. To quantify this effect we used the Large Velocity Gradient (LVG) approximation for CS. We assumed a line width of $0.6 \mathrm{~km}^{-1}$ and a column density of $10^{12} \mathrm{~cm}^{-2}$ (optically thin case). We adopted the collisional rates $\mathrm{CS} / \mathrm{p}-\mathrm{H}_{2}$ provided by Denis-Alpizar et al. (2018). We obtain $T_{\mathrm{ex}}(J=1-0)=7.3 \mathrm{~K}$ and $T_{\mathrm{ex}}(J=2-1)=3.9 \mathrm{~K}$. If the collisional rates $\mathrm{CS} / \mathrm{o}-\mathrm{H}_{2}$ from the same authors are adopted, then the derived excitation temperatures for these transitions are 8.9 and $4.9 \mathrm{~K}$, respectively. From the observed line parameters of the $J=1-0$ transition of $C^{34} S$ and ${ }^{13} \mathrm{C}^{34} \mathrm{~S}$ given in Table A.1, we derive $\mathrm{N}\left(\mathrm{C}^{34} \mathrm{~S}\right)=6.5 \times 10^{12}$ $\mathrm{cm}^{-2}$, and $\mathrm{N}\left({ }^{13} \mathrm{C}^{34} \mathrm{~S}\right)=5.5 \times 10^{10} \mathrm{~cm}^{-2}$. These values are a factor 2.2 and 2.6 lower than those provided in Table 3 , respectively, which have been obtained assuming a uniform rotational temperature of $10 \mathrm{~K}$. Hence, the main source of uncertainty in the derived column densities could be related to the assumption of a uniform rotational temperature for all rotational levels of a linear molecule (LTE conditions), rather than to the adopted rotational temperature.

\section{Appendix B: Observed line parameters and frequency predictions up to $J=\mathbf{3 0}$}

The observed line parameters for the sulfur-bearing molecules discussed in this paper are given in Table A.1. For $\mathrm{HC}_{3} \mathrm{~S}^{+}$they are given in Table 1 . Frequency predictions for $\mathrm{HC}_{3} \mathrm{~S}^{+}$up to $J=$ 30 are given in Table B.1 
Table B.1. Predicted line frequencies for $\mathrm{HC}_{3} \mathrm{~S}^{+}$.

\begin{tabular}{|c|c|c|c|c|c|}
\hline$J_{\mathrm{u}}-J_{1}$ & $v(\mathrm{MHz})$ & $E_{\mathrm{u}}(\mathrm{K})$ & $A_{\mathrm{ul}}\left(\mathrm{s}^{-1}\right)$ & $S_{i j}$ & $g_{\mathrm{u}}$ \\
\hline $1-0$ & $5470.9255 \pm 0.0004$ & 0.3 & $1.901 \times 10^{-9}$ & 1 & 3 \\
\hline $2-1$ & $10941.8469 \pm 0.0008$ & 0.8 & $1.825 \times 10^{-8}$ & 2 & 5 \\
\hline $3-2$ & $16412.7601 \pm 0.0011$ & 1.6 & $6.601 \times 10^{-8}$ & 3 & 7 \\
\hline $4-3$ & $21883.6608 \pm 0.0013$ & 2.6 & $1.623 \times 10^{-7}$ & 4 & 9 \\
\hline $5-4$ & $27354.5451 \pm 0.0014$ & 3.9 & $3.241 \times 10^{-7}$ & 5 & 11 \\
\hline $6-5$ & $32825.4087 \pm 0.0016$ & 5.5 & $5.687 \times 10^{-7}$ & 6 & 13 \\
\hline $7-6$ & $38296.2475 \pm 0.0024$ & 7.4 & $9.131 \times 10^{-7}$ & 7 & 15 \\
\hline $8-7$ & $43767.0575 \pm 0.0036$ & 9.5 & $1.374 \times 10^{-6}$ & 8 & 17 \\
\hline $9-8$ & $49237.8344 \pm 0.0057$ & 11.8 & $1.970 \times 10^{-6}$ & 9 & 19 \\
\hline $10-9$ & $54708.5742 \pm 0.0084$ & 14.4 & $2.716 \times 10^{-6}$ & 10 & 21 \\
\hline $11-10$ & $60179.2727 \pm 0.0118$ & 17.3 & $3.631 \times 10^{-6}$ & 11 & 23 \\
\hline $12-11$ & $65649.9258 \pm 0.0160$ & 20.5 & $4.731 \times 10^{-6}$ & 12 & 25 \\
\hline $13-12$ & $71120.5294 \pm 0.0211$ & 23.9 & $6.034 \times 10^{-6}$ & 13 & 27 \\
\hline $14-13$ & $76591.0793 \pm 0.0270$ & 27.6 & $7.556 \times 10^{-6}$ & 14 & 29 \\
\hline $15-14$ & $82061.5714 \pm 0.0340$ & 31.5 & $9.315 \times 10^{-6}$ & 15 & 31 \\
\hline $16-15$ & $87532.0017 \pm 0.0420$ & 35.7 & $1.133 \times 10^{-5}$ & 16 & 33 \\
\hline $17-16$ & $93002.3659 \pm 0.0512$ & 40.2 & $1.361 \times 10^{-5}$ & 17 & 35 \\
\hline $18-17$ & $98472.6599 \pm 0.0615$ & 44.9 & $1.618 \times 10^{-5}$ & 18 & 37 \\
\hline $19-18$ & $103942.8796 \pm 0.0731$ & 49.9 & $1.906 \times 10^{-5}$ & 19 & 39 \\
\hline $20-19$ & $109413.0209 \pm 0.0860$ & 55.1 & $2.226 \times 10^{-5}$ & 20 & 41 \\
\hline $21-20$ & $114883.0796 \pm 0.1003$ & 60.7 & $2.580 \times 10^{-5}$ & 21 & 43 \\
\hline $22-21$ & $120353.0517 \pm 0.1161$ & 66.4 & $2.969 \times 10^{-5}$ & 22 & 45 \\
\hline $23-22$ & $125822.9330 \pm 0.1335$ & 72.5 & $3.396 \times 10^{-5}$ & 23 & 47 \\
\hline $24-23$ & $131292.7193 \pm 0.1524$ & 78.8 & $3.862 \times 10^{-5}$ & 24 & 49 \\
\hline $25-24$ & $136762.4066 \pm 0.1730$ & 85.3 & $4.368 \times 10^{-5}$ & 25 & 51 \\
\hline $26-25$ & $142231.9907 \pm 0.1954$ & 92.2 & $4.917 \times 10^{-5}$ & 26 & 53 \\
\hline $27-26$ & $147701.4674 \pm 0.2196$ & 99.2 & $5.510 \times 10^{-5}$ & 27 & 55 \\
\hline $28-27$ & $153170.8328 \pm 0.2457$ & 106.6 & $6.149 \times 10^{-5}$ & 28 & 57 \\
\hline $29-28$ & $158640.0825 \pm 0.2737$ & 114.2 & $6.836 \times 10^{-5}$ & 29 & 59 \\
\hline $30-29$ & $164109.2126 \pm 0.3038$ & 122.1 & $7.572 \times 10^{-5}$ & 30 & 61 \\
\hline
\end{tabular}

